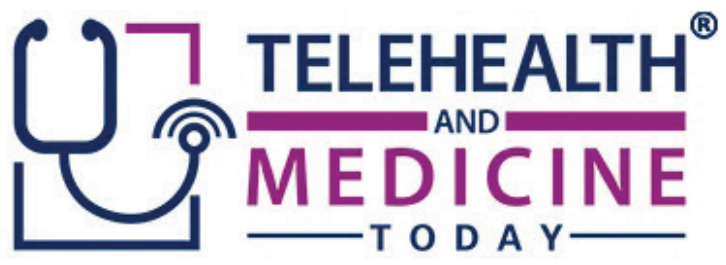

\title{
Tele-Rehabilitation for Individuals with Multiple Sclerosis and Mobility Disorders-A Feasibility and Pilot Study
}

Heather Barksdale, PT, DPT, NCS, MSCS ${ }^{1}$, Brittany McHugh, PT, DPT, MSCS $^{1}$, Wayne Hodges, RN, PMD, SCRN ${ }^{2}$, Jessica Peters, RN, PMD, SCRN ${ }^{3}$, Carmen Smotherman, MS, MPH${ }^{4}$, Paul M. Hoffman, MD²

Affiliations: ${ }^{1}$ UF Health Jacksonville Rehabilitation, Jacksonville, FL, USA; ${ }^{2}$ UF Health Jacksonville Neuroscience Institute, Jacksonville, FL, USA; ${ }^{3}$ UF Health Jacksonville, Jacksonville, FL, USA;

${ }^{4}$ Center for Data Solutions, University of Florida College of Medicine, Jacksonville, FL, USA

Corresponding Author: Heather Barksdale, Email: heather.barksdale@jax.ufl.edu

Keywords: Multiple Sclerosis, Physical Therapy, Telehealth, Tele-Rehab, Tele-Rehabilitation

Section: Original Clinical Research

Objective: To determine the feasibility of conducting an 8-week physical therapist (PT)guided tele-rehabilitation (TR) program for persons with multiple sclerosis (MS) and mobility deficits.

Design, setting, and participants: This feasibility study had a pre-post-intervention, non-randomized design. Nine subjects with a confirmed diagnosis of MS without a clinical exacerbation in the last year were selected from the MS Center of Excellence at UF Health Jacksonville (UFHJ). Inclusion criteria included demonstrated mobility deficits on initial examination, basic computer competency, and a home with high-speed internet connection. Examinations were performed face-to-face, pre-post-intervention in the Neurology Research Clinic at UFHJ.
TR was delivered to the patient's homes in Florida and South Georgia.

Intervention: An informed consent was obtained prior to initial examination. Selfreported measures included quality of life (QOL) and fatigue. Mobility measures included timed 10-m walk, five times sit to stand, and Berg balance scale. Following inclusion, home safety was evaluated and computer equipment was installed. TR intervention utilized the Jintronix ${ }^{\circledR}$ web-based platform system with a kinetic camera that demonstrated selected exercises by an avatar, allowing for real-time feedback on the quality of performance as well as tracking of adherence. The individualized exercises were assigned by the evaluating $P T$ based on the initial assessment. Subjects participated in weekly TR visits with the 
prescribing PT to further monitor and adjust the therapy program.

Main outcome measures: Primary outcome measures were patient satisfaction, safety, and compliance. Secondary outcome measures were projected travel cost, self-reported fatigue and QOL, and mobility testing measures. To compare the TR effect on mobility, a comparator persons with MS group was selected retrospectively. Eight persons with MS referred from the MS Center at UFHJ for outpatient (OP) PT from January 2018 through September 2019 were identified by UFHJ electronic database search for ICD 10 code G35. The inclusion criteria for mobility deficits and functional measurements were the same as those in TR group.

Results: Eight subjects completed the TR program with no adverse events. They reported satisfaction, demonstrated compliance, and saved $\$ 8,487$ in projected travel costs. All TR subjects noted improvement in self-reported fatigue, QOL, or mobility measures. Improvement in mobility measures had wide variability but did not differ between TR and OP groups.

Conclusions: The 8-week PT-monitored TR program was shown to be feasible, safe, and well received by persons with MS. Larger studies focused on specific dysfunction leading to mobility deficits and longer intervention times will be necessary to determine efficacy.

\section{BACKGROUND}

Multiple sclerosis (MS) is a multifocal disease of the central nervous system, often producing variable and long-standing symptoms that may lead to disability, including loss of mobility. Diminished mobility not only reduces functional independence but can also lead to obesity and cardiovascular disease associated with a sedentary lifestyle. Furthermore, mobility deficits and increased risk of falling are identified in approximately $75 \%$ of persons with MS. ${ }^{1}$ The goal of neuro-rehabilitation often focuses on the reduction of impairments affecting function, activities of daily living, personal activities, and social participation in order to allow for increased independence along with improvement in disability level and quality of life (QOL). ${ }^{1-3}$

There is limited efficacy for disease-modifying therapy (DMT) to prevent the accumulation of long-term disability in MS. ${ }^{4}$ Only two DMTs have demonstrated a significant effect on the progressive phases and accumulated disability in MS., 5 Evidence demonstrates the benefits of exercise training for persons with MS for improvement in walking, fatigue, cognition, fitness, anxiety, and depression. ${ }^{3-4,7-9}$ Studies have also found that exercise training can result in lower annual relapse rates for persons with MS compared with a control group..$^{10}$ Therefore, utilization of neuro-rehabilitation may be an adjunctive strategy to reduce exacerbations, improve disease management, and slow accumulated disability in MS.

Although studies reveal the importance of incorporating a multidisciplinary approach of care for persons with MS, including rehabilitation, barriers including coverage and access to highly trained specialists continue to persist. Access is further limited for persons with mobility deficits, who either live in remote areas or do not have access to transportation services. Tele-rehabilitation (TR) may provide an option for these individuals to access important rehabilitation services.

The recent coronavirus (COVID-19) pandemic highlights the need for improved options in access to rehabilitation services. The Centers for Medicare and Medicaid Services (CMS) recently made temporary exceptions to providers who can be included in reimbursement for telehealth services, including physical therapist (PT) 
delivered by two-way, real-time interactive communication between the patient at home and provider at a distant site. ${ }^{11}$

Tele-video and distance technology are shown to be feasible and well received by persons with mobility disorders, including MS in Veteran's Administration studies. ${ }^{12,13}$ However, studies specific to the use of TR continue to be limited. A literature review published in $2019^{14}$ cited a total of six articles published on the use of TR for persons with MS. One study examined the effect on upper extremity function, ${ }^{15}$ whereas the remaining studies examined the effect on lower extremity function. ${ }^{12,16-19}$ None of the lower extremity studies examined the effect on QOL, fatigue, and mobility measures in the same individuals. Furthermore, only one study included a virtual live component to the TR program or an intervention-based comparator group. ${ }^{19}$

The purpose of this study was to explore the feasibility of delivering home-based, PT-guided TR to persons with MS and mobility deficits. In addition, we provided data on the effect size and variability of mobility measures, which might be useful for future studies of efficacy for the use of PT-guided TR. An outpatient (OP) treatment group was selected retrospectively to compare the groups' mobility outcome scores, as well as to suggest guidelines for the delivery mode of rehabilitation most appropriate for individual patients. These results will help to determine the selection of persons with MS for specific mobility deficits, group size, and length of intervention necessary to demonstrate efficacy. The intervention portion of this study was completed prior to the beginning of the COVID-19 pandemic.

\section{METHODS AND FINDINGS}

\section{TR Intervention}

Subjects were recruited from the MS Center of Excellence at UF Health Jacksonville (UFHJ) from among 350 persons with MS being followed. The project was approved by the University of Florida Institutional Review Board (study \#201702552). Funding was provided by a Dean's Grant program from the College of Medicine, University of Florida, Jacksonville, United States. Nine subjects with a confirmed diagnosis of MS were enrolled (six subjects with relapsing MS with disability and three subjects with primary progressive MS). All were clinically stable without a documented clinical exacerbation within the last year. All subjects demonstrated mobility deficits that included medium to high fall risk on the Berg balance scale (BBS), below normal performance on the five times sit-to-stand test (5XSTS), or a gait speed below normal on the $10-\mathrm{m}$ walk test (10MWT). All enrolled subjects continued current disease modifying therapy. However, those who were actively taking Ampyra (dalfampridine) were excluded due to the possible effect on ambulation scores during the study. ${ }^{20,21}$ Candidates were also excluded if they demonstrated inability to perform five unassisted sit-to-stand exercises from a chair with handles, inability to ambulate $10 \mathrm{~m}$ with an assistive device and without physical aide, inability to safely maintain sitting balance without physical assist, or if findings on a mini mental state exam (MMSE) were consistent with moderate-to-severe dementia. Ability to communicate in English, to view a computer-based system (appropriate visual acuity with correction if needed), access to a home, high-speed internet connection, and basic computer competency were required.

After consenting to participate, an initial face-toface assessment was performed by a boardcertified neurologist at the UFHJ Neurology Research Clinic to determine if the subjects met the inclusion criteria and had no other neurological deficits that would interfere with the functional measures. One of the two boardcertified MS-specialized PTs at UFHJ evaluated 
lower extremity strength (manual muscle testing), reflexes, tone (spasticity), ${ }^{22}$ proprioception, light touch sensation and coordination in order to establish baseline, and need for initiation of exercise program. Functional evaluation included the 10MWT, ${ }^{23,24}$ 5XSTS, ${ }^{25}$ and BBS. ${ }^{26,27}$ QOL and fatigue were both measured by self-reported questionnaire. QOL was assessed on the MS Impact Scale $(\text { MSIS-29) })^{28}$ and fatigue measured on the Modified Fatigue Impact Scale. ${ }^{29}$ The neurological assessment included a standard neurological assessment and MMSE.

Once initial assessment was complete, the nine subjects were contacted by one of the two participating study registered nurses ( $\mathrm{RNs}$ ) to schedule in-home installation of the TR equipment. Subjects resided in rural and urban areas of Florida and South Georgia. Computer equipment used was a common, off-the-shelf gaming computer system with a kinetic camera and a web camera. The study RN installed the equipment and assessed the safety of the exercise area to used. Fall and trip hazards were removed, and confirmation of adequate room space and lighting was ensured. The RN then instructed the subject (and caregiver if available) on the use of the equipment. A "cheat sheet" guide was also provided to the subjects, including utilization instructions, log-in instructions, and contact numbers for communicating with the RNs to trouble shoot connection difficulties if they were to occur.

A personalized exercise program was prescribed by the evaluating PT based on the initial face-toface assessment. Each program was designed to address the impairments and mobility deficits identified at assessment. The exercises were also designed to address an individual's balance status for safety. Therefore, exercises were prescribed either in sitting or standing and modified to include handhold assist when needed. Each subject was prescribed between six and eight initial exercises to mirror a typical outpatient home program. The computer setup facilitated use of the Jintronix ${ }^{\circledR}$ web-based platform. ${ }^{30-32}$ The platform allowed the treating PT to select, assign, and monitor frequency of equipment usage and performance of exercises. It also provided demonstration of the selected exercises by an avatar, which the subjects would then mimic for performance and repetition. The program and kinetic tracking system further provided feedback to the subjects on quality of motion and speed of task. Subjects were required to perform the exercise program at least twice a week. However, they had access to the program throughout the week and were advised to perform as much as desired unless fatigue, spasticity, or pain worsened.

Once weekly, one of the two MS-certified PTs participated in a live, webcam-based TR session to remotely observe the subjects' performance of the exercise program and to make modifications of the program as needed. These modifications included adjustments to the difficulty of the exercises, type of exercise, or adjustment of number of repetitions as needed. Within 2 weeks of finishing the 8-week TR program, each subject returned to the clinic for the same face-to-face assessment performed initially.

\section{Comparator Outpatient Subjects}

Following the completion of the intervention portion of the study, a comparator OP rehabilitation group (OP) was selected to retrospectively compare the effect of PT on mobility between the groups. Subjects with a confirmed diagnosis of MS seen at the UFHJ OP rehabilitation clinic from January 2018 through September 2019 were identified through an electronic database search for ICD 10 code G35. All subjects selected were seen by the same PTs 
who had also performed the evaluation and treatment for the TR group and met the same inclusion criteria for mobility dysfunction as those used to select TR. Demographic information on each group is shown in Table 1.

The chart review included scores for testing, including lower extremity strength (manual muscle testing), reflexes, tone (spasticity), proprioception, and light touch sensation and coordination. Functional evaluation included the 10MWT, 5XSTS, and BBS. Data were then reviewed for effects on mobility. Data were summarized using means and standard deviations. Differences from pre- to postintervention were analyzed using the Signed rank test, and the differences between changes in the two groups were analyzed using the Wilcoxon rank sum test. The level of significance was set at 0.05 .

\section{RESULTS}

\section{Feasibility}

Eight of the nine enrolled TR subjects completed the 8 -week program. The one subject who did not complete the intervention was dropped due to the reported and continued difficulty with the use of technology. No adverse events, falls, or injuries were noted during or following the completion of the program. Following the completion of the study, all TR subjects also completed a phone survey. The survey reviewed the subjects' perception of feasibility, barriers to use, and travel time saved by using the TR program compared with attending $\mathrm{OP}$ rehabilitation. The overall quality and value of the TR experience was highly rated by all participants. Specifically, training in the use of the Jintronix ${ }^{\circledR}$ program, ease in operation of the equipment, and availability and feedback from the weekly observations by the PT were reported as excellent.

Review of the projected travel cost for the eight subjects was determined by GPS distance from home to UFHJ in miles multiplied by $\$ 0.56$ (standard mileage reimbursement rate) and amounted to a combined patient travel savings of $\$ 8,487.23$ for eight weekly in-person appointments with a MS-certified PT. No show rate was also compared between groups. Whereas no visits were missed (by cancelation or no show) in the TR group, a total of six visits were missed in the OP group.

\section{Function}

All TR subjects demonstrated an improvement in at least one mobility score that met the established minimum detectable change (MDC) ${ }^{33-35}$ (Table

2). Comparisons of pre- and post-intervention

Table 1. Demographic Information.

\begin{tabular}{l|c|c|c|c|c|} 
Variable & \multicolumn{1}{c}{ Category } & \multicolumn{1}{c|}{ TR } & OP Group & Overall & P-Value \\
Group & $6(75)$ & $7(88)$ & $13(81)$ & $0.999 *$ \\
\hline Gender & F & $6(13)$ & $3(19)$ & \\
\hline
\end{tabular}

*Fisher's exact test

Variable Group
\begin{tabular}{|l|c|c|c|c|c|c|c|c|c|c|} 
N & Mean & $\begin{array}{c}\text { Standard } \\
\text { Deviation }\end{array}$ \\
\hline Age & TR & 8 & 55.63 & 10.29 & 40 & 47.5 & 57 & 62 & 72 & $0.205^{* *}$ \\
\hline & OP & 8 & 49.13 & 11.78 & 33 & 36.5 & 55 & 57.5 & 62 & \\
\hline
\end{tabular}

**Wilcoxon's Rank Sum Test 


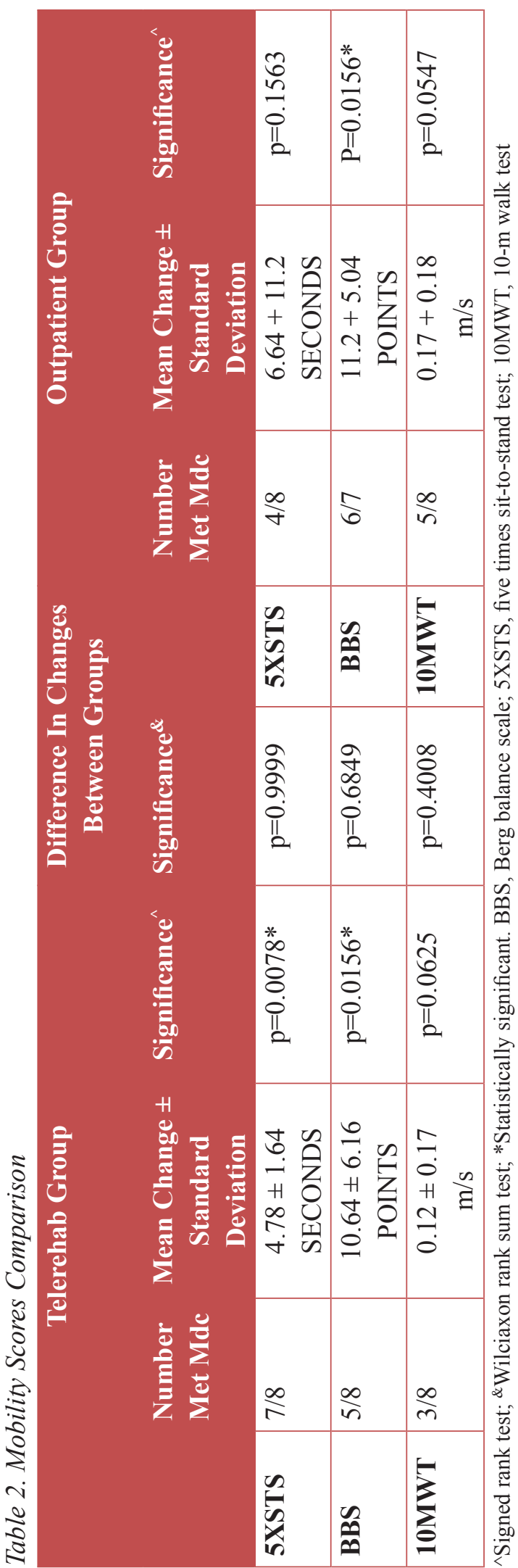

scores showed statistically significant improvement for 5XSTS and BBS (Table 2). Self-reported fatigue and QOL scores were also reviewed. All subjects reported improvement in self-reported fatigue. Six out of eight subjects also reported improvement in self-reported QOL (Table 3).

The comparator OP group demonstrated either improvement or stability following an 8-week PT intervention. All but one subject demonstrated an improvement in at least one mobility score that met the MDC. However, only BBS scores were found to have a statistically significant change (Table 2). When TR was compared with OP, no statistically significant differences were found between changes in mobility scores for either 10MWT, 5XSTS, or BBS (Table 2).

\section{DISCUSSION}

Previous studies demonstrate the positive effect that rehabilitation can have on mobility and QOL for persons with MS. However, very few studies have examined the use of TR in this population. The unique aspects of the MS disease process require ongoing and specialty care to best prepare the individual for the changing symptomology and progression of the disease. This often includes mobility deficits, worsening fatigue, and changes to QOL. These individuals also often have difficulty with transportation or access to skilled therapy services in their area. Furthermore, the recent COVID-19 pandemic highlights the need for alternative modes of delivery for persons who are in need of rehabilitation. TR offers the opportunity to decrease the travel time and cost. While this study required specific computer system installation for participants, future TR setups may include the use of patient's home devices, therefore decreasing or eliminating installation cost. TR also provides an opportunity for improved access to therapists with specialized 
Table 3: Quality of Life and Fatigue Scores

\begin{tabular}{|l|c|c|c|}
\multicolumn{2}{c}{$\begin{array}{c}\text { Number With Self } \\
\text { Reported Improvement }\end{array}$} & $\begin{array}{c}\text { Mean Change } \pm \text { Standard } \\
\text { Deviation }\end{array}$ & Significance $^{\wedge}$ \\
\hline MSIS-29 & $6 / 8$ & $14.75 \pm 21.81$ & $\mathrm{p}=0.0859$ \\
\hline Fatigue Scale & $8 / 8$ & $\begin{array}{c}14.75 \pm 9.59 \\
\text { POINTS }\end{array}$ & $\mathrm{p}=0.0078^{*}$ \\
& & & \\
\hline
\end{tabular}

Signed rank test; *Statistically significant

training or advanced certifications. The lower no show rate in the TR group can be explained by the convenience of performing TR in the home instead of traveling to a distant site. However, another contributing factor may be in the use of the Jintronix ${ }^{\circledR}$ system. A previous study on the use of gaming in conjunction with rehabilitation reported that "key factors" in game design, including choice, reward, and goals, lead to increased motivation and engagement. ${ }^{36}$

While this study was underway, another TR study was published. ${ }^{37}$ That pilot study also examined the feasibility and use of TR for persons with MS. Overall findings mirrored that findings for this study in that TR were found to be feasible and there were no significant differences between the TR and OP groups for the functional outcome measures examined. However, the dosage of intervention, method of administration, and patient selection between studies were different. Furthermore, both studies included the use of the BBS to assess balance and fall risk. However, initial mean pre-intervention score for this study was $32.5 / 56$ compared with $43.25 / 56$ in the other, ${ }^{37}$ indicating lower starting mobility for this subject population.

This study included a weekly live PT-guided TR session with the use of the Jintronix ${ }^{\circledR}$ platform to assist with biofeedback. Subjects were also required to perform the exercises using only the Jintronix ${ }^{\circledR}$ platform at least one additional session a week. All subjects performed the exercises between three and five times per week pointing to increased adherence and compliance with the exercise program.

Selection guidelines for TR for the neurological population have yet to be established. We are not aware of any studies to date comparing the change in mobility scores for persons with MS between TR and OP groups, looking specifically at mobility related to transfer. Although this study did not demonstrate a statistical difference in change in mobility scores between groups, it did identify possible trends that could facilitate future selection of patients for the use of TR. One such comparison was noted when examining how many subjects in each group demonstrated improvement in their mobility scores to meet the established MDC. While seven of the eight subjects in the TR group met the MDC for the 5XSTS score, only four of the eight subjects demonstrated that improvement in the OP group. Conversely, the subjects in the comparator group showed only statistically significant improvement in BBS scores. This suggests that although no statistical difference was noted between groups, patients in the TR group seemed to improve more with the functional task of sit to stand while the OP group did not. In this feasibility and exploratory study, the number of patients in each group was small and variations in pre-intervention scores were large. These trends, therefore, should be viewed with caution but are consistent with the MDC results for each group. 
The findings may also be related to the specific interventions performed in each setting. For example, the TR group performed tasks often in the sitting or stationary stance position, while the OP group often performed more dynamic standing balance tasks and less tasks in a sitting position. Prior research has pointed to the importance of task specificity in the neurological patient and these findings support that concept. This study may assist clinicians in future selection of persons for TR. For example, if a person with MS is having difficulty with sitting balance and transfers, TR may be indicated. However, if a person with MS is having more difficulty with dynamic standing balance and ambulation, the OP setting may be more appropriate. Often, clinicians select TR services for individuals who are more independent due to the possible safety concerns. However, these findings also suggest that persons with increased mobility deficits may benefit from improvement not only in mobility but also in self-perceived QOL and fatigue. All TR subjects noted a reduction in fatigue that met statistical significance $(p=0.003)$, while six of eight subjects noted an improved QOL that did not meet the statistical significance $(p=0.097)$. This can be attributed to the wide variation in selfrating of improvement in this small group of participants.

A possible limitation in this study was due to the Jintronix ${ }^{\circledR}$ program, providing a limited list of exercises for the PT to utilize when developing the exercise programs. The Jintronix ${ }^{\circledR}$ program only allowed the PT to modify repetitions or timeframes required to complete tasks, which could have hindered adequate rehab progressions for participants with higher mobility function at baseline. Future development of impairmentspecific exercises may further improve subject mobility deficits. Subjects participated in TR and OP for eight weeks. This may have limited the total dosage and may not have been a sufficient time frame to ascertain the level of improvement that would otherwise be identified.

The average direct cost savings for each of the eight persons with MS in the TR group was $\$ 1,060$ (906 euros). The number varied by distance traveled and was based upon driving in a personally owned vehicle at the standard mileage reimbursement rate $(\$ 0.56$ per mile, 0.43 euros). No additional costs incurred while traveling, such as food or lodging, were considered. The study was not designed to compare the patient travel cost savings to additional costs of providing the TR to the patient. However, no additional PT staff was required for the evaluation of TR visits, and each case was performed in lieu of a 45-min in-person OP visit.

Each patient required two round trips of the $\mathrm{RN}$ for the installation of equipment and patient/caregiver training, and retrieval of equipment at the end of the study period. Travel expenses (mileage) would be equivalent to 2 patient days of in-person rehab. RN salary was partially offset by combining travel with normal duties of one RN (outreach to smaller hospitals and EMS agencies in the region). This study provided evidence of what the training needs of the patient/caregiver should cover and common issues with operation during study. It is felt that the instillation could be performed by a well-trained technician such as a PT assistant, emergency medical technician, or licensed practical nurse. With the onset of COVID-19, home safety evaluations are now being conducted with the utilization of TR, reducing travel cost to the provider. Furthermore, system installation was required due to software requirements of the Jintronix ${ }^{\circledR}$ system and desire for standardization across subjects in this 
feasibility study. Utilization of existing patient-owned devices and more portable versions of the Jintronix ${ }^{\circledR}$ platform are being developed to reduce costs and increase accessibility.

Readiness for the use of telemedicine has been recognized as a problem for older adults predominantly owing to inexperience with use of technology. ${ }^{38}$ The importance of computer literacy for patient selection was seen in one subject who dropped out of the study due to difficulty with the utilization of the computer system. The subject and caregiver were both trained on the utilization of the system initially, but the subject required frequent over the phone/ webcam instructions and corrections on the use of the system, all of which were clearly described on the "cheat sheet."

\section{CONCLUSIONS}

This study indicates that TR is a feasible and safe mode of delivery for individuals with MS. With proper installation and home safety evaluation, patients or their caregivers with a moderate level of computer use knowledge were very compliant and receptive of rehabilitation delivered on a game-based program in conjunction with PT monitoring. The results also suggest that TR has the potential to be an effective method of rehabilitation to improve patient-perceived fatigue, QOL, and deficits related to reduced mobility. Future studies with a more specific focus on type of mobility deficit, larger number of subjects in each group, and longer interventions are needed to confirm the efficacy of TR for specific mobility deficits in persons with MS.

Funding Statement: Funding was provided by a Dean's Grant program from the College of Medicine, University of Florida, Jacksonville. The funders had no role in study design, data collection and analysis, decision to publish, or preparation of the manuscript.

Conflicts of Interest: The authors declare no conflicts of interest.

Contributors: This study design was an adaptation of a face-to-face home TR study initiated and developed at the VA Rural Health Resource Center Resource Center-Eastern Region (PMH) and promoted nationally as a Promising Practice by the Office of Rural Health, Department of Veterans Affairs. The University of Florida College of Medicine-Jacksonville Dean's grant application and manuscript preparation were joint efforts by $\mathrm{PMH}, \mathrm{HB}$, and WH. All investigators participated in the review and editing of the manuscript. TR subject selection, neurological evaluations, and cognitive screening were performed by PMH. Pre- and post-intervention PT evaluation and tele-video visits were performed by $\mathrm{HB}$ and BM. OP group selection and previous treatment had been performed by $\mathrm{HB}$ and BM. Equipment procurement, installation, and home safety evaluations were performed by WH and JP. Statistical analysis was performed by CS. The Departments of Neurology (PMH) and Center for Data Solutions (CS) University of Florida College of Medicine-Jacksonville, Neuroscience Institute (PMH, WH, and JP), and UFHJ Rehabilitation (HB and $\mathrm{BM}$ ) contributed investigator time to the study.

\section{REFERENCES}

1. Lord SE, Wade DT, Halligan PW. A comparison of two physiotherapy treatment approaches to improve walking in multiple sclerosis: A pilot randomized controlled study. Clin Rehabil. 1998;12:477-86.

2. Haselkorn JK, Hughes C, Rae-Grant A, et al. Summary of comprehensive systematic review: Rehabilitation in multiple sclerosis: Report of the guideline development, 
dissemination, and implementation subcommittee of the American Academy of Neurology. Neurology. 2015;85(21):1896-903.

3. Brichetto G, Rinaldi S, Spallarossa P, et al. Efficacy of physical therapy in multiple sclerosis as measured with the modified fatigue impact scale and ambulation index: A retrospective study. NeuroRehabilitation. 2013;33:107-12.

4. Edwards T, Pilutti LA. The effect of exercise training in adults with multiple sclerosis with severe mobility disability: A systematic review and future research directions. Mult Scler Relat Disord. 2017;16:31-9.

5. Montalban X, Hauser S, Kappos L, et al. Ocrelizumab versus placebo in primary progressive multiple sclerosis. $N$ Engl $J$ Med. 2017;376(3):209-20.

6. Kappos L, Bar-Or A, Cree BAC, et al. Siponimod versus placebo in secondary progressive multiple sclerosis (EXPAND): A double blind, randomized, phase 3 study. Lancet. 2018;391(10127):1263-73.

7. Aminian S, Ezeugwu VE, Motl RW, et al. Sit less and move more: Perspectives of adults with multiple sclerosis. Disabil Rehabil. 2019;41(8):904-11.

8. Coote S, Uszynski M, Herring MP, et al. Effect of exercising at minimum recommendations of the multiple sclerosis exercise guideline combined with structured education or attention control education-Secondary results of the step it up randomised controlled trial. $B M C$ Neurology. 2017; 17:119.

9. Kubsik-Gidlewska AM, Klimkiewicz P, Klimkiewicz R, et al. Rehabilitation in multiple sclerosis. Adv Clin Exp Med. 2017;26(4):709-15.

10. Dalgas U. Exercise therapy in multiple sclerosis and its effects on function and the brain. Neurodegenerative Dis. Manag. 2017;7(6s):35-40.

11. Centers for Medicare \& Medicaid Services. Physicians and other clinicians: CMS flexibilities to fight COVID-19. April 29, 2020 [cited 2020 May 20]; 1-13. Available from: https://www.cms.gov/files/document/ covid-19-physicians-and-practitioners.pdf

12. Finkelstein $\mathrm{J}$, Lapshin $\mathrm{O}$, Castro $\mathrm{H}$, et al. Home-based physical telerehabilitation in persons with multiple sclerosis: A pilot study. JRRD. 2008;45(9):1361-74.

13. Hoffman PM, McCoy SC. Increasing access to care for ALS and MS Veterans in rural areas. PVA Summit and Expo. 2014: (session 1417). Las Vegas, NV.

14. Yeroushalmi S, Maloni H, Costello K, Wallin M. Telemedicine and multiple sclerosis: A comprehensive literature review. J Telemed Telecare. 2019;0(0):1-14.

15. Huijgen B, Vollenbroek-Hutten $M$, Zampolini M, et al. Feasibility of a homebased telerehabilitation system compared to usual care: Arm/hand function in patients with stroke, traumatic brain injury and multiple sclerosis. $J$ Telemed Telecare. 2008;14(5):249-56.

16. Conroy S, Zhan M, Culpepper W, et al. Self-directed exercise in multiple sclerosis: Evaluation of a home automated telemanagement system. $J$ Telemed Telecare. 2018;24(6):410-19.

17. Paul L, Coulter EH, Miller L, et al. Webbased physiotherapy for people moderately affected with Multiple Sclerosis:

Quantitative and qualitative data from a randomized, controlled pilot study. Clin Rehabil. 2014;28(9):924-35.

18. Tallner A, Streber R, Hentschke C, et al. Internet-supported physical exercise training for persons with multiple sclerosis: A randomized, controlled study. Int $\mathrm{J} \mathrm{Mol}$ Sci. 2016;17(10):E1667.

19. Ortiz-Guiterrez R, Cano-de-la-Cuerda R, Galan-del-Rio F, et al. A telerehabilitation program improves postural control in multiple sclerosis patients: A Spanish preliminary study. Int J Env Res Pub He. 2013;10(11):5697-710.

20. Malhotra M, Ghai P, Narasimhan B, et al. Dalfampridine: Review on its recent development for symptomatic improvement in persons with multiple sclerosis. Arabian J Chem. 2016;9:S1443-9. 
21. Applebee A, Goodman AD, Mayadev AS, et al. Effects of dalfampridine extended-release tablets on 6-minute walk distance in persons with multiple sclerosis: A post hoc analysis of a double-blind, placebo-controlled trial. Clin Therapeut. 2015;37(12):2780-7.

22. Barnes MP, Kent RM, Semlyen JK, et al. Spasticity in multiple sclerosis. Neurorehabilitation and Neural Repair. 2003;17(1):66-70.

23. Dalgas U, Severinsen K, Overgaard K. Relations between 6 minute walking distance and 10 meter walking speed in persons with multiple sclerosis and stroke. Arch Phys Med Rehabil. 2012;93:1167-72.

24. Rossier P, Wade D. Validity and reliability comparison of 4 mobility measures in patients presenting with neurologic impairment. Arch Phys Med Rehabil. 2001;82:9-13.

25. Møller A, Bibby BM, Skjerbæk A, et al. Validity and variability of the 5-repetition sit-to stand test in persons with multiple sclerosis. Disabil Rehabil. 2012;34(26):2251-8.

26. Toomey E, Coote S. Between-rater reliability of the 6-minute walk test, berg balance scale, and handheld dynamometry in people with multiple sclerosis. Int J MS Care. 2013;15:1-6.

27. Nilsagard Y, Lundholm C, Denison E, et al. Predicting accidental falls in people with multiple sclerosis-A longitudinal study. Clin Rehabil. 2009;23:259-69.

28. Hobart J, Lamping D, Fitzpatrick R, et al. The Multiple Sclerosis Impact Scale (MSIS-29): A new patient-based outcome measure. Brain. 2001;124:962-73.

29. Flachenecker P, Kumpfel T, Kallmann B, et al. Fatigue in multiple sclerosis: A comparison of different rating scales and correlation to clinical parameters. Mult Scler. 2002;8:523-6.

30. Archambault P, Nahid GN, Kairy D, et al. Toward establishing clinical guidelines for an arm rehabilitation virtual reality system. Biosystems Biorobotics. 2014;7:263-70.

31. Lauze M, Martel DD, Agnoux A, et al. Feasibility, acceptability and effects of a home-based exercise program using a gerontechnology on physical capacities after a minor injury in community-living older adults: A pilot study. J Nutr Health Aging. 2016;1:1-10.

32. Lauze M, Martel DD, \& Aubertin-Leheudre M. Feasibility and effects of a physical activity program using gerontechnology in assisted living communities for older adults. JAMDA. 2017;1:1-7.

33. Learmonth Y, Paul L, McFadyen A, et al. Reliability and clinical significance of mobility and balance assessments in multiple sclerosis. Int J Rehabil Res. 2012;35(1):69-74.

34. Barhuly A, Bohannon R, Gorack W. Gait speed is a responsive measure of physical performance for patients undergoing short-term rehabilitation. Gait Posture. 2012;36:61-4.

35. Goldberg A, Chavis M, Watkins J, Wilson T. The five-times-sit-to-stand test: Validity, reliability and detectable change in older females. Aging Clin Exp Res. 2012;24(4):339-44.

36. Lohse K, Shirzad N, Verster A, et al. Video games and rehabilitation: Using design principles to enhance engagement in physical therapy. JNPT. 2013;37:166-75

37. Fjeldstad-Pardo C, Thiessen A, Pardo G. Telerehabilitation in multiple sclerosis: Results of a randomized feasibility and efficacy pilot study. Int $J$ Telerehabil. 2018;10(2):55-64.

38. Covinsky KE, Shi Y, Lu AD, Lam K. Assessing telemedicine unreadiness among older adults in the United States during the COVID-19 pandemic. JAMA Intern Med. 2020;E1-E3.

Copyright Ownership: This is an open access article distributed in accordance with the Creative Commons Attribution Non Commercial (CC BY-NC 4.0) license, which permits others to distribute, adapt, enhance this work non-commercially, and license their derivative works on different terms, provided the original work is properly cited and the use is non-commercial. See: http:// creativecommons.org/licenses/by-nc/4.0. 
